\title{
Increased myofiber remodelling and NFATc1-myonuclear translocation in rat postural skeletal muscle after experimental vestibular deafferentation
}

\author{
Nicholas Luxa ${ }^{\mathrm{a}, \mathrm{b}}$, Michele Salanova ${ }^{\mathrm{a}, \mathrm{b}}$, Gudrun Schiffl ${ }^{\mathrm{a}, \mathrm{b}}$, Martina Gutsmann ${ }^{\mathrm{a}, \mathrm{b}}$, Stéphane Besnard ${ }^{\mathrm{c}}$, \\ Pierre Denise $^{\mathrm{c}}$, Andrew Clarke ${ }^{\mathrm{d}}$ and Dieter Blottner ${ }^{\mathrm{a}, \mathrm{b}, *}$ \\ ${ }^{a}$ Department of Vegetative Anatomy, Neuromuscular Group, Charité - University Medicine Berlin, Berlin, \\ Germany \\ ${ }^{\mathrm{b}}$ Center for Space Medicine Berlin (ZWMB), Berlin, Germany \\ ${ }^{c}$ Physiology Lab, UCBN, UFR Medicine, Caen Cedex, France \\ ${ }^{\mathrm{d}}$ CBF, ENT Department, Charité - University Medicine Berlin, Berlin, Germany
}

Received 28 February 2013

Accepted 4 October 2013

\begin{abstract}
.
BACKGROUND: The vestibular system undergoes considerable modification during spaceflight [5]. This is paralleled by microgravity-induced muscle atrophy [6]. However, the possibility of vestibulo-autonomic regulatory mechanisms affecting skeletal muscle structure and function have not yet been addressed.

OBJECTIVE: We hypothesise that the vestibular system affects anti-gravitational skeletal muscle phenotype composition, size and the transcriptional factor called nuclear factor of activated T cells (NFATc1).

METHODS: In a laboratory study, we examined the morphological and histochemical properties including intramyocellular NFATc1 changes in slow-type soleus muscle of chemically labyrinthectomized rats (VLx; $n=8$ ) compared to a control group (Sham; $n=6$ ) after a period of one month.

RESULTS AND CONCLUSION: Neurochemical vestibular deafferentation resulted in smaller myofibre sizes, altered myofibre phenotype composition, high yields of hybrid fibre formation, and reduced myonuclear NFATc1 accumulation as signs of slowtype myofibre atrophy, myofibre type remodelling, and altered nuclear transcriptional activity in the postural soleus muscle of rats. We propose that vestibulo-autonomic modification of skeletal muscles occurs during prolonged microgravity. Our findings are likely to have implications for vestibular rehabilitation in clinical settings.
\end{abstract}

Keywords: Labyrinthectomy (vestibular lesion), skeletal muscle soleus, NFATc1 (NFAT2)

${ }^{*}$ Corresponding author: Dieter Blottner, Charité - University Medicine Berlin, Department of Vegetative Anatomy, Neuromuscular Group, Philippstrasse 12, D-10115 Berlin, Germany. Tel.: +49 30450528 412; Fax: +49 30450528 954; E-mail: dieter. blottner@charite.de.

\section{Introduction}

Muscle atrophy induced by inactivity during prolonged spaceflight is a well-established phenomenon [6]. In addition, the absence of gravity results in adaptation in the vestibular system, primarily due to the loss of the prevalent gravitational stimulus to 
the otolith apparatus [7]. This loss is paralled by dicordant visual, haptic and/or proprioceptive sensations generating a sensory integration conflict [8]. During the initial days in microgravity it is understood that the vestibular afferents are reweighted in the associated brainstem circuitry countering this sensory conflict $[4$, $5,8]$. In the central nervous system this has consequences for sensory-motor coordination and those cognitive processes associated with spatial orientation [5, 8]. The influence of tonic vestibulospinal modulation of motor activity on the structure of anti-gravitational muscles, such as the calf soleus has previously been reported [21]. Furthermore, interaction between the vestibular system and regulatory autonomic mechanisms has also been demonstrated [26]. Accordingly, these vestibulo-autonomic regulatory mechanisms are likely to be similarly modified in microgravity, and in turn, affect muscle function during the duration of spaceflight.

Skeletal muscle contributes approximately $40 \%$ of body weight and consists of different fibre types, which express various specific isoforms or types of myosin heavy chains (MyHC), detectable by immunohistochemistry: Type I (slow MyHC positve), type II (fast MyHC positive) and hybrid fibres (fast $\mathrm{MyHC}$ and slow MyHC positive) $[16,19]$. The anti-gravitational soleus of the rat is composed of these major fibre types, i.e. predominantly slow MyHC, a minor proportion of fast MyHC2A and a lesser proportion of fast $\mathrm{MyHC2X}$ [20].

We have recently demonstrated that the information from the otolith organs has a direct influence on bearing bone density and bone remodeling, probably mediated by modulation of the sympathetic tone after bilateral labyrinthectomy in rats [14]. In a number of ground-based animal studies, using surgical bilateral labyrinthectomy in neonatal ferrets or adult rats, significant changes in the slow-type muscle soleus were observed $[10,21]$. Other reports confirmed that vestibulosympathetic reflex activity exerts a strong influence on the vegetative system with regard to maintenance of postural blood pressure control $[15,26]$. However, the signaling pathways underlying the changes observed in the slow-type soleus muscle in this experimental model remained unknown.

In the present approach we hypothesise that the absence of vestibular afferent input to postural skeletal muscle, as induced by chemical labyrinthectomy in rats [25], affects myofibre size and type composition in a slow-type soleus muscle; whereby hybrid fibre formation (expressing both slow and fast-type MyHC) is regarded as a reliable sign of structural and functional myofibre remodelling. More specifically, we employed the intramyocellular myonuclear vs cytosolic shuttle mechanism of the transcriptional nuclear factor of activated T cells (NFATc1) as a reliable biomarker; (i) this represents a key factor in the subcellular signalling pathway involved in activity-dependent slow type myosin switching [13] and (ii) also acts as a nerve activity sensor [24].

\section{Methods}

\subsection{Animal experiment}

After two weeks of vivarium acclimatization fourteen $(n=14)$ adult female Sprague-Dawley rats of 3 months of age and weighing between $285 \pm$ $20 \mathrm{~g}$ (JANVIER, France) were divided into two experimental groups. Animals of the VLx-group underwent chemical labyrinthectomy after global anesthesia by trans-tympanic injection of sodium arsanilate (Darsenilic $5 \mathrm{mg}$ in $0.1 \mathrm{ml}$, SIGMA ALDRICH) in each ear (VLx, $n=8$ ). Control rats received trans-tympanic injections of saline solution (Sham, $n=6$ ). Volatile anaesthesia was initiated in a sealed chamber by $3,5 \%$ Isoflurane (flow rate: $21 / \mathrm{min}$ ) and maintained by $2 \%$ Isoflurane (flow rate: $0,81 / \mathrm{min}$ ) for the duration of the intervention. This procedure was followed by adequate analgesia by means of pro-paracetamol (prodafalgan, MERCK).

Experiments were conducted in accordance with the European Community Council Directive 86/6609/ EEC. The protocol was approved by our regional ethical committee (Comité d'Ethique Normandie en Matière d'Expérimentation Animale, CENOXEMA, number assigned 0412-01). The animals were housed group-wise (2-3 individuals) in standard type III $\mathrm{H}$ cages of $820 \mathrm{~cm}^{2} \times 18 \mathrm{~cm}$ at $21^{\circ} \mathrm{C}+1 /-1^{\circ} \mathrm{C}$ with a 12:12 h light-dark cycle (light period between 8:00 and 20:00 h) with food and water freely available.

Clinical assessment of successful bilateral labyrithectomy was performed by a previously validated clinical vestibular scale [3]. Arsanilate injection induced peripheral vestibular hair cells loss and to a lesser degree cochlear hair cells loss without damaging the vestibular nerve or nuclei [25].

After a period of one month the animals were sacrificed by administration of $5 \mathrm{ml}$ intraperitoneal barbituric administration $(150 \mathrm{mg} / \mathrm{kg})$ and the complete soleus muscle was dissected, immediately frozen 
into liquid nitrogen, and stored at $-80^{\circ} \mathrm{C}$ for subsequent morphological and immunohistochemical analyses. Transversal cryosections of approximately 8$10 \mu \mathrm{m}$ thick were prepared from the middle portion of the muscle using a cryostat (Kryostat Frigocut 2800 E, Reichert-Jung, Germany) set between $-24^{\circ} \mathrm{C}$ and $-20^{\circ} \mathrm{C}$.

\subsection{Immunohistochemistry}

The myofibre phenotype composition, size and NFATc1-accumulation were assessed by immunolabeling with primary antibodies and secondary antibodies labelled with fluorochromes. Cryosections mounted on slides were fixed with $4 \%$ formaldehyde, washed in trishydroxymethylaminomethan (TRIS)-buffered saline and permeabilized in $0,3 \%$ Triton X-100. Triple staining was applied on fixed cryosections for confocal laser microscopy analysis, including primary antibodies versus a membrane marker, dystrophin (SC 15376, Santa Cruz Biotechnology), slow MyHC (M8421, Sigma) and fast MyHC (M4276, Sigma). Primary antibodies were diluted 1:100-1:1000. Alexa Fluor (Molecular Probes) $635 \mathrm{~nm}, 555 \mathrm{~nm}$ and $488 \mathrm{~nm}$ were employed as secondary antibodies at 1:500-1:2000 dilution. Controls include incubation of cryosections lacking the primary or secondary antibody and showed negative results.

The staining protocol for epifluorescence microscopy contained NFATc1 (Santa Cruz Biotechnology), dystrophin (Sigma), fast MyHC (Sigma), and 4',6diamine-2-phenylindole dihydrochloride (DAPI) enabling detection of intracellular myonuclei of fast MyHC negative fibres and an evaluation of the myonuclear NFATc1-accumulation. Primary antibodies were diluted 1:100-1:1000. Alexa Fluor (Molecular Probes) 635,555 and $488 \mathrm{~nm}$ were also employed here as secondary antibodies at 1:1000-1:2000 dilution.

\subsection{Myofibre phenotype composition and cross-sectional area (CSA)}

Myofibres from randomly selected areas of the cryosection sets were counted and classified for phenotype composition by anti-MyHC immunohistochemistry. Thus, slow MyHC (type I, red), fast MyHC (type II, green) and dystrophin (blue) were visualized using a high signal resolution confocal laser scanning microscope (Leica TCS SP-2 with Leica confocal software version 2.61 build 1537; Leica Micosystems Heidelberg GmbH, Germany) equipped with a three-channel multilaser system (argon laser, 458$514 \mathrm{~nm}$; helium/neon laser, $543 \mathrm{~nm}$; helium/neon laser, $633 \mathrm{~nm}$ ). Standardized laser scanning presettings (i.e., single scan mode, $1024 \times 1024$ scan format, $25 \%-$ $50 \%$ laser intensities, $500 \pm 10.0 \mathrm{~V}$ gain settings) were used.

For myofibre CSA measures the scanned digitized images of triple-immunostained cryosections were processed by the proprietary software package (Leica Microsystems). On the basis of dystrophin-immunolabeled outer membrane borders, we determined the pixel area $\left(\mu \mathrm{m}^{2}\right)$ of given oval/circle planes that matched with the cross-sectioned myofibre profiles of slow MyHC positiv, fast MyHC positive, and hybrid fibres (fast MyHC and slow MyHC positive). Details of the morphometry procedure were previously reported [2]).

\subsection{Evaluation of myonuclear NFAT-accumulation}

A meandering screening pattern was applied for arbitrary myonuclei detection. The immunostained cryosections were photographed under a fluorescence microscope (Zeiss Axioplan) at a magnification of $\times 1000$ with a Visitron Systems Cool Snap digital camera using appropriate software (Metavue Software 7.6.0.0; MDS Analytical Technologies). The photographed nuclei were counted and the subcellular localisation of NFATc1 was evaluated. Dystrophin membrane marker visualisation enables differentiation between intranuclear, extranuclear nuclei or nuclei from satellite cells attached to the outer fibre membranes. Myonuclei of fast MyHC positive fibres were excluded. Immunohistochemical intensities less than cytosol NFATc1 immunofluorescence or cytosol-like intranuclear NFATc1 accumulation were classified as NFATc1 myonuclei translocation negative, whereas increased levels of immunoreactivity reflecting intranuclear NFATc1 accumulation were classified as NFATc1 myonuclei translocation positive.

\subsection{Statistics}

Statistical analysis was performed using PASW Statistics 18 and Sigma Plot 9.0. Pearson's ChiQuadrat-Test was used to test for significant differences in myonuclear NFAT-accumulation and myofibre phenotype composition; the Students t-test for unpaired values was used to test CSA determinations. The significance level was set to $P<0.001$. 

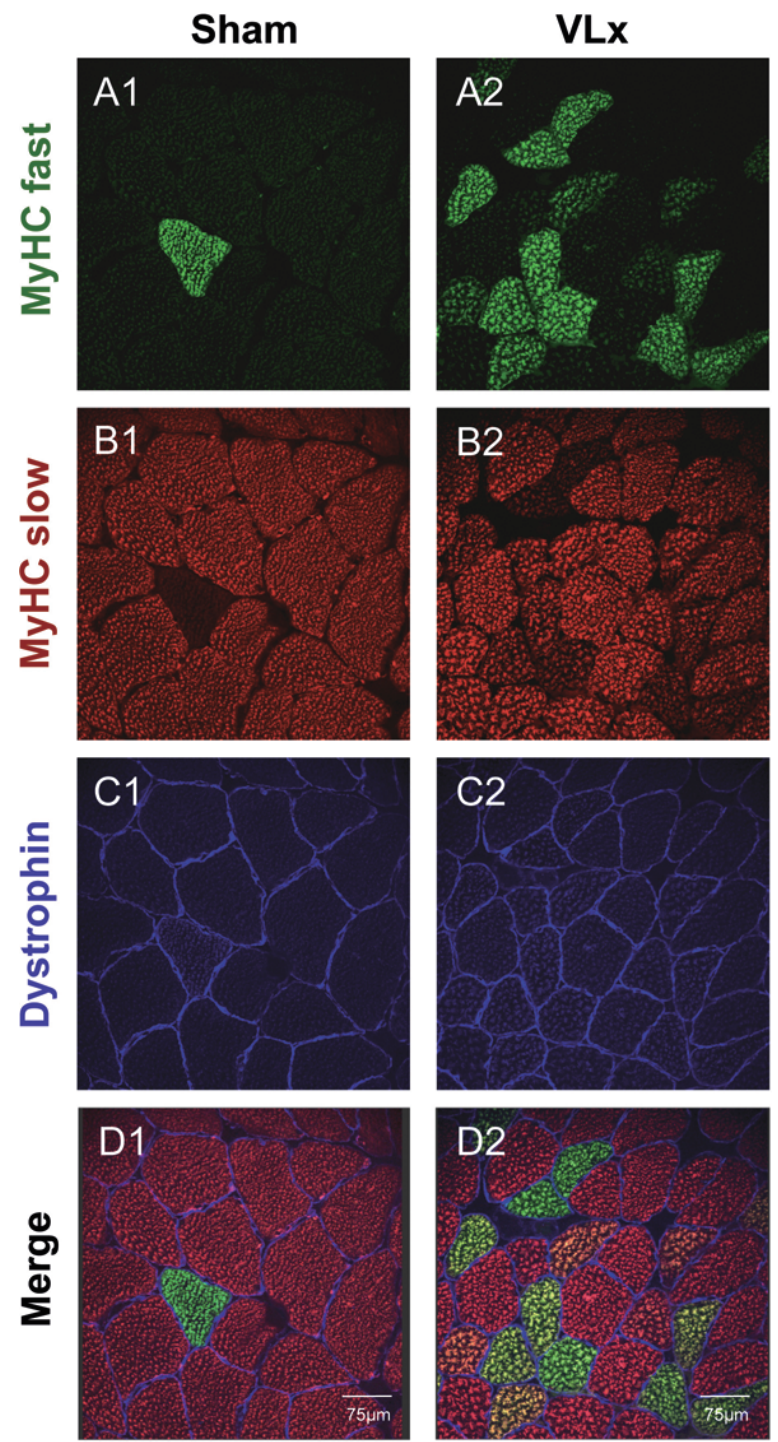

Fig. 1. Representative morphological and histochemical changes in slow soleus muscle of chemically labyrinthectomized rats (VLx) compared to a control group (Sham) after a period of one month. Cross sections after immunhistochemistry of fast $\mathrm{MyHC}$ (A1 and $\mathrm{A} 2$ ), slow MyHC (B1 and B2), dystrophin (C1 and C2) and merge (D1 and D2).

\section{Results}

\subsection{Myofibre phenotype composition}

The myofibre phenotype of rat soleus muscle in the VLx group and in the Sham group was examined after a period of one month, with the focus on type I (slow MyHC positve), type II (fast MyHC positive) and hybrid fibres (fast MyHC and slow MyHC positive) (Fig. 1). Fibre-type distribution counting showed

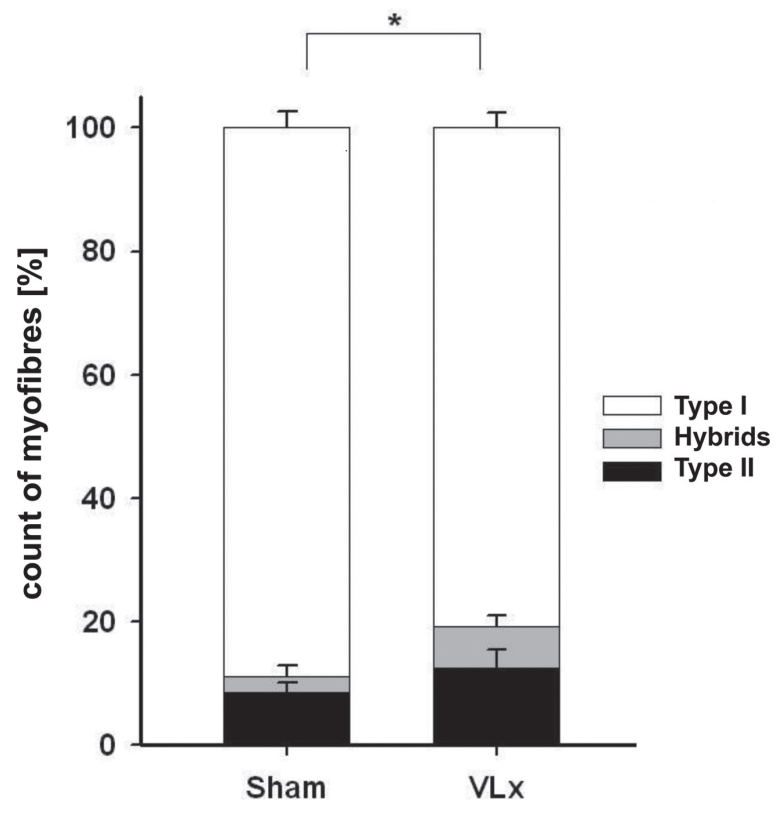

Fig. 2. Myofibre Phenotype Composition (M. soleus; 14 animals; 3180 counted myofibres). Error bars show standard error of the mean (SEM). Chi-Quadrat-Test of Pearson revealed high significant change $(* P<0.001)$ for myofibre phenotype composition distribution for VLx vs. Sham.

Table 1

Cross-sectional area of myofibre type I, myofibre type II and hybrid Fibres. The results are expressed in their average affected by their standard error (means \pm SEM). Students t-test for unpaired values revealed high significant change $\left({ }^{*} P<0,001\right)$ of cross-sectional area for type I myofibres

\begin{tabular}{lcc}
\hline Group & Sham & VLx \\
\hline number of measured fibres & 1183 & 1997 \\
CSA myofibre type I $\left[\mu \mathrm{m}^{2}\right]$ & $2514 \pm 30$ & $* 2030 \pm 19$ \\
CSA myofibre type II $\left[\mu \mathrm{m}^{2}\right]$ & $1488 \pm 53$ & $1504 \pm 29$ \\
CSA hybrid fibres $\left[\mu \mathrm{m}^{2}\right]$ & $1463 \pm 102$ & $1414 \pm 49$ \\
\hline
\end{tabular}

a highly significant change in the composition of all myofibre phenotypes in the VLx group $(80,7 \%$ myofibre type I; $12,4 \%$ myofibre type II; $6,9 \%$ hybrids), in comparison to the Sham group (88,9\% myofibre type I; $8,6 \%$ myofibre type II; 2,5\% hybrids) (Fig. 2).

\subsection{Cross-sectional area}

The CSAs of rat soleus muscle fibres in the two groups were measured after a period of one month, considering the phenotype of the myofibres (Fig. 3). A highly significant reduction in the CSA ( $-19 \%$ vs. sham) of slow MyHC positive muscle fibres, assumed to be type I myofibres, was observed in the VLx group compared to the Sham group (see Table 1). 


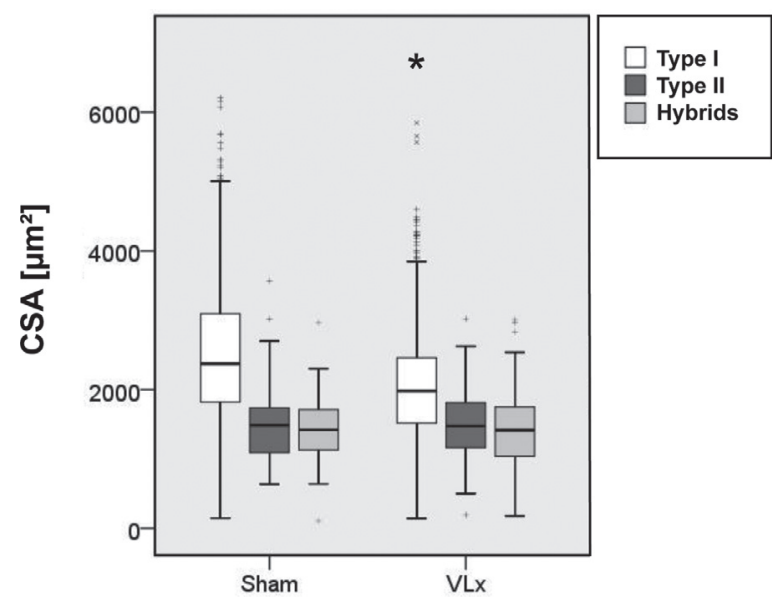

Fig. 3. Cross-sectional area of myofibre phenotypes (M. soleus; 14 animals; 3180 measured fibres). Box-whisker-plots show distribution of cross-section area within the individuals. Box show interquartils range (IQR); + Outliers $(1,5 \mathrm{x} \mathrm{IQR}<\mathrm{CSA}<3 \mathrm{x} \mathrm{IQR})$; $\mathrm{x}$ extreme value $(\mathrm{CSA}>3 \mathrm{x} \mathrm{IQR})$. Students t-test for unpaired values revealed high significant change $\left({ }^{*} P<0.001\right)$ of cross-sectional area for type I myofibres.

\subsection{NFATc1 accumulation}

The subcellular localisation of NFATc1 was examined, focusing on the dephosporylated nuclear NFATc1, which impresses as intranuclear focal fluorescence (see legend of Fig. 4). Morphological analyses of NFATc1 nuclei accumulation revealed a highly significant reduction of NFATc1 in the myofibre nuclei of fast MyHC negative muscle fibres, assumed to be type I myofibres (NFATc1 positive myonuclei versus cytosolic expression level: VLx group 42,0\%; Sham group 50,7\%) (see Fig. 4).

\section{Discussion}

The present findings indicate that neurochemical vestibular deafferentation induces pronounced slow myofibre atrophy, elevated levels of myofibre type re-distribution and remodelling in the postural slowtype rat soleus muscle. More importantly, further morphological signs of altered intramyocellular NFATc1 translocation (myofibre biomarker of nucleocytoplasmic shuttling and transcriptional activity) now showed clear evidence for extensive myofibre remodelling particularly of the slow MyHC-immunoreactive myofibers in the postural soleus muscle in our VLx rat model.

Shall et al. [21] reported a detailed analysis of the developmental MyHC content of soleus muscle in an-

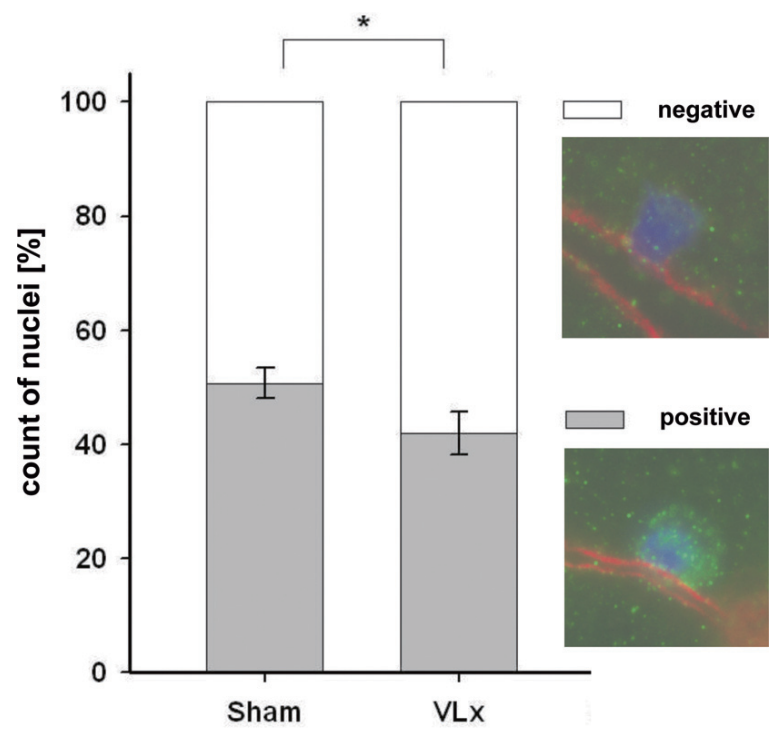

Fig. 4. NFATc1 myonuclear accumulation ( $n=14$ animals; 2432 nuclei total counts). Error bars show standard error (SEM). ChiQuadrat-Test of Pearson revealed high significant change $\left({ }^{*} P<\right.$ 0.001) of NFAT accumulation distribution for VLx vs. Sham. Legend pictures show a representation of a myonucleus with lacking and/or cytosol-like NFATc1 accumulation and a myonucleus with intra- and perinuclear NFATc1 accumulation. 1000x magnification ("digitally" enlarged). Fluorochrome labels: DNA (blue), dystrophin (red), and NFATc1 (green).

other small vertebrate (ferret). 120 days after birth the ferrets showed less slow MyHC, more fast MyHC2A, and significantly more fast MyHC2X following surgical neonatal bilateral labyrinthectomy [21]. As in our VLx rats, the type I myofibres of the matured labyrinthectomized ferrets were smaller than those of the control group, thus supporting our proposal of a neurovestibular control of the slow-type myofibre size and phenotype in vertebrate anti-gravitational skeletal muscle.

In contrast to the reduced type I myofibre CSA rate found in the present study, Kasri et al. [10] reported no changes in the CSA of any fibre types in soleus muscle of VLx rats. Apart from obvious methodological differences (surgical vs. chemical VLx ablation) this may also be the result of incomplete compensation in the vestibular nuclei, leading to the described changes in antigravitional skeletal muscles of experimental small vertebrate animals.

However, neurons in the vestibular nuclei apparently regain spontaneous activity quickly after bilateral vestibular neurectomy, indicative of compensatory mechanisms [27]. In the absence of vestibular afferents non-labyrinthine inputs from the skin, muscles of the neck, limb and trunk as well as the viscera 
continue to be integrated in the vestibular nuclei and cerebellum [27] and play a more predominant role in signalling body position in space. Consequently, vestibulo-spinal and reticulo-spinal postural reflexes involving anti-gravitational muscles can remain functional subsequent to peripheral vestibular lesion. On the other hand, ground-based laboratory experiments have demonstrated an increase in muscle sympathetic nerve activity after activation of the otolith organs [17, 22 ] in the human and a modulation of sympathetic tone at several spinal levels in the cat $[11,12]$. On the basis of the present findings and the cited literature we propose that altered levels of vestibulo-sympathetic activation represent a link between the vestibular system and anti-gravitational muscles. However, the vestibular adaptation process might fail to compensate this after bilateral labyrinthectomy, whether performed in ground-based studies or under true microgravity conditions.

Vestibular dysfunction can also lead to hyperactivity in experimental animals [1,3,23]. Indeed, rats showed some hyperactivity one month after vestibular lesioning, which might have affected the experimental outcome of the present study. In contrast Shall et al. [21] reported unchanged motor activity, while Kasri et al. [10] described a reduction of locomotor movements after surgical bilateral labyrinthectomy. As already mentioned, such discrepancies between studies could be due to the different species, protocols and procedures of performing the in-vivo lesion.

Our results are in accordance with the previously described atrophy patterns and slow-to-fast myofibre transition observed under microgravity conditions [6] and with more recent data from a 91-day spaceflight animal experiment in mice [18]. Differential expression and nuclear translocation of the signalling nuclear marker NFATc1 found in the present study are clear signs of the slow myofibre atrophy, thus providing closer insights into the transcriptional pathway controlling skeletal muscle fibre type $[9,19]$. Significantly higher amounts of hybrid fibres, i.e. evidence of myofibre type remodelling or switch, was confirmed in the soleus muscle of the rat after bilateral labyrithectomy in the present study as previously shown [10]. Thus, there is now clear evidence of a lower transcription of slow MyHC in VLx that might result in maladaptation of the soleus muscle myofibre composition resulting in impaired postural muscle functions.

In conclusion, the present study demonstrates that elimination of vestibular input in the VLx rat, an accepted ground-based animal model of vestibular deaf- ferentation [25], resulted in considerable structural, as well as molecular signalling changes, particularly in slow-type soleus skeletal muscle fibres and their myonuclei; thus supporting the concept of neurovestibular control mechanisms of postural skeletal muscles. However the specific neural pathways underlying such vestibulo-muscle interactions remain unknown. Further experiments are required to rule out any potential interference related to the vestibular lesion paradigm in the animal model. These include observations of longterm effects as well as detailed analysis of the associated neural circuitry and behavioural changes. The VLx rat model used in our study is likely to produce higher alterations than a microgravity environment due to the complete deafferentation of the labyrinths - a condition not taking place in spaceflight.

Nevertheless suppression of vestibular information to skeletal muscle, in particular to the slow-type myofibre phenotype control, as found in the VLx rat in ground-based experiments might well serve as a model to further study the intricate functional convolution between microgravity-induced reductions in vestibular neural activity and skeletal muscle atrophy during spaceflight. The present findings contribute to the increasing body of evidence demonstrating the relationship between behavioural, or system physiology and molecular mechanisms, and improve our understanding, both of basic gravitational physiology and associated clinical disorders.

\section{Acknowledgements}

This work was supported by grants from the Deutsches Zentrum für Luft-und Raumfahrt (DLR), Germany (\# 50WB0821 to D. B.), the Centre National d'Etudes Spatiales (CNES), France (to S. B. and P.D.) and ESA/ISGP/ISSBB/ELGRA (to N.L.).

\section{References}

[1] S. Besnard, M.L. Machado, G. Vignaux, M. Boulouard, A. Coquerel, V. Bouet, T. Freret, P. Denise and V. LelongBoulouard, Influence of vestibular input on spatial and nonspatial memory and on hippocampal NMDA receptors, Hippocampus 22 (2012), 814-826.

[2] D. Blottner, N. Serradj, M. Salanova, C. Touma, R. Palme, M. Silva, J.M. Aerts, D. Berckmans, L. Vico, Y. Liu, A. Giuliani, F. Rustichelli, R. Cancedda and M. Jamon, Morphological, physiological and behavioural evaluation of a 'Mice in Space' housing system, Journal of Comparative Physiology B 179 (2009), 519-533. 
[3] P. Boadas-Vaello, J. Riera and J. Llorens, Behavioral and Pathological Effects in the Rat Define Two Groups of Neurotoxic Nitriles, Toxicological Science 88 (2005), 456-466.

[4] A.H. Clarke and L. Kornilova, Ocular torsion response to active head-roll movement under one-g and zero-g conditions, Journal of Vestibular Research 17 (2007), 99-111.

[5] G. Clément, M. Reschke and S. Wood, Neurovestibular and Sensorimotor Studies in Space and Earth Benefits, Current Pharmaceutical Biotechnology 6 (2005), 267-283.

[6] R.H. Fitts, D.R. Riley and J.J. Widrick, Functional and structural adaptations of skeletal muscle to microgravity, Journal of Experimental Biology 204 (2001), 3201-3208.

[7] A. Graybiel, Significance of vestibular organs in problems of weightlessness, Life Sciences and Space Research 1 (1963), 19-32.

[8] M. Heer and W.H. Paloski, Space motion sickness: Incidence etiology, and countermeasures, Autonomic Neuroscience: Basic and Clinical 129 (2006), 77-79.

[9] G. Hogan, L. Chen, J. Nardone and A. Rao, Transcriptional regulation by calcium, calcineurin, and NFAT, Genes and Development 17 (2003), 2205-2232.

[10] M. Kasri, F. Picquet and M. Falempin, Effects of unilateral and bilateral labyrinthectomy on rat postural muscle properties: the soleus, Experimental Neurology 185 (2004), 143153.

[11] I.A. Kerman, R.M. McAllen and B.J. Yates, Patterning of sympathetic nerve activity in response to vestibular stimulation, Brain Research Bulletin 53 (2000), 11-16.

[12] I.A. Kerman and B.J. Yates, Regional and functional differences in the distribution of vestibulosympathetic reflexes, American Journal of Physiology 275 (1998), R824-R835.

[13] H.P. Kubis, R.J. Scheibe, J.D. Meissner, G. Hornung and G. Gros, Fast-to-slow transformation and nuclear import/export kinetics of the transcription factor NFATc1 during electrostimulation of rabbit muscle cells in culture, Journal of Physiology 541(3) (2002), 835-847.

[14] R. Levasseur, J.P. Sabatier, O. Etard, P. Denise and A. Reber, Labyrinthectomy decreases bone mineral density in the femoral metaphysis in rats, Journal of Vestibular Research $\mathbf{1 4}$ (2004), 361-365.

[15] H. Normand, O. Etard and P. Denise, Otolithic and tonic neck receptors control of limb blood flow in humans, Journal of Applied Physiology 82 (1997), 1734-1738.

[16] D. Pette and R.S. Staron, Transition of muscle fiber pheno- typic profiles, Histochemistry and Cell Biology 115 (2001), 359-372.

[17] C.A. Ray, Interaction between vestibulosympathetic and skeletal muscle reflexes on sympathetic activity in humans, Journal of Applied Physiology 90 (2001), 242-247.

[18] D. Sandonà, J.F. Desaphy, G.M. Camerino, E. Bianchini, S. Ciciliot, D. Danieli-Betto, G. Dobrowolny, S. Furlan, E. Germinario, K. Goto, M. Gutsmann, F. Kawano, N. Nakai, T. Ohira, Y. Ohno, A. Picard, M. Salanova, G. Schiffl, D. Blottner, A. Musarò, Y. Ohira, R. Betto, D. Conte and S. Schiaffino, Adaptation of mouse skeletal muscle to long-term microgravity in the MDS mission, PLoS One 7 (2012), e33232.

[19] S. Schiaffino, Fibre types in skeletal muscle: a personal account, Acta Physiologigica 199 (2010), 451-463.

[20] A.L. Serrano, M. Murgia, G. Pallafacchina, E. Calabria, P. Coniglio, T. Lømo and S. Schiaffino, Calcineurin controls nerve activity-dependent specification of slow skeletal muscle fibers but not muscle growth, Proc Natl Acad Sci USA 98 (2001), 13108-13113.

[21] M.S. Shall, D.J. Lanzino, S.V. Cleave and S.J. Goldberg, Neonatal bilabyrinthectomy leads to changes in skeletal muscle fiber form and function, Journal of Vestibular Research $\mathbf{1 5}$ (2005), 253-262.

[22] T.L. Shortt and C.A. Ray, Sympathetic and vascular responses to head-down neck flexion in humans, American Journal of Physiology 272 (1997), 1780-1784.

[23] P.F. Smith, T. Brandt, M. Strupp, C.L. Darlington and Y. Zheng, Balance before reason in rats and humans, Ann NY Acad Sci 1164 (2009), 127-133.

[24] J. Tothova, B. Blaauw, G. Pallafacchina, R. Rudolf, C. Argentini, C. Reggiani and S. Schiaffino, NFATc1 nucleocytoplasmic shuttling is controlled by nerve activity in skeletal muscle, Journal of Cell Science 119 (2006), 1604-1611.

[25] G. Vignaux, C. Chabbert, S. Gaboyard-Niay, C. Travo, M.L. Machado, P. Denise, F. Comoz, M. Hitier, G. Landemore, B. Philoxène and $\mathrm{S}$. Besnard, Evaluation of the chemical model of vestibular lesions induced by arsanilate in rats, Toxicology and Applied Pharmacology 258 (2012), 61-71.

[26] B.J. Yates, Vestibular influences on the sympathetic nervous system, Brain Research Reviews 17 (1992), 51-59.

[27] B.J. Yates and D.M. Miller, Integration of nonlabyrinthine inputs by the vestibular system: role in compensation following bilateral damage to the inner ear, Journal Vestibular Research 19 (2009), 183-189. 